\title{
Three-dimensional photoacoustic imaging of subcutaneous microvasculature in vivo
}

Hao F. Zhang, Meng-Lin Li, Konstantin Maslov, George Stoica, Lihong V. Wang

Hao F. Zhang, Meng-Lin Li, Konstantin Maslov, George Stoica, Lihong V. Wang, "Three-dimensional photoacoustic imaging of subcutaneous microvasculature in vivo," Proc. SPIE 6086, Photons Plus Ultrasound: Imaging and Sensing 2006: The Seventh Conference on Biomedical Thermoacoustics, Optoacoustics, and Acousto-optics, 60861E (6 March 2006); doi: 10.1117/12.645138

SPIE. Event: SPIE BiOS, 2006, San Jose, California, United States 


\title{
Three-dimensional photoacoustic imaging of subcutaneous microvasculature in vivo
}

\author{
Hao F. Zhang ${ }^{\mathrm{a}}$, Meng-Lin $\mathrm{Li}^{\mathrm{a}}$, Konstantin Maslov ${ }^{\mathrm{a}}$, George Stoica ${ }^{\mathrm{b}}$, Lihong V. Wang ${ }^{\mathrm{a} *}$ \\ ${ }^{a}$ Dept. of Biomedical Engineering, Texas A\&M University, College Station, TX, USA 77843-3120; \\ ${ }^{b}$ Dept. of Veterinary Pathobiology, Texas A\&M University, College Station, TX, USA 77843-5547
}

\begin{abstract}
Three-dimensional photoacoustic imaging of subcutaneous microvasculature in small animals is realized in vivo using photoacoustic microscopy. Previously, degraded lateral resolution in the out-of-focus region made three-dimensional visualization impractical. With the help of a virtual-detector synthetic aperture focusing technique, depth-independent lateral resolution is achieved. Therefore, volumetric imaging of the vessels is realized with a lateral resolution of $45 \mu \mathrm{m}$ and an axial resolution of $15 \mu \mathrm{m}$. Detailed structural information, such as vessel depth and spatial orientation, are revealed with new clarity.
\end{abstract}

Keywords: Photoacoustic, microscopy, microvasculature

\section{INTRODUCTION}

How to image the three-dimensional (3D) morphology of subcutaneous blood vessels noninvasively is a challenging problem. First, the small diameter of the blood vessels, ranging from $20 \mu \mathrm{m}$ to several hundred micrometers, requires an imaging modality that has high spatial resolution. Second, such an imaging modality must be sensitive to either hemoglobin concentration or blood flow in order to separate the blood vessels from the surrounding soft tissues. Third, this imaging modality must have enough penetration depth to image the major vasculature lying beneath the dermal layer (1 3 mm deep from the skin surface in a small animal). Finally, this modality must be able to acquire volumetric data.

Given the abovementioned requirements, optical-absorption contrast-based photoacoustic microscopy (PAM) ${ }^{1}$ is an ideal tool for this purpose. PAM is a hybrid imaging technique that detects absorbed diffuse photons ultrasonically based on the photoacoustic (PA) effect ${ }^{2}$. The spatial resolution of PAM is determined by the central frequency, bandwidth, and numerical aperture (NA) of the ultrasonic detector. When an ultrasonic transducer with high central frequency, wide band, and larger NA is used, PAM can achieve high spatial resolution imaging by localizing the PA sources with high resolution. Because oxyhemoglobin and deoxyhemoglobin are two of the dominating optical absorbers within the visible spectral range, PAM is highly sensitive to blood and, consequently, blood vessels. When working at an isobestic point, where these two forms of hemoglobin have identical optical absorption, PAM functions as a blood detector to image total hemoglobin concentration. Relying on ultrasonic localization, PAM can penetrate deeper than traditional highresolution optical imaging modalities and still retain high spatial resolution because ultrasonic scattering is 2-3 orders of magnitude weaker than optical scattering ${ }^{3}$. Moreover, the PA waves are produced by absorbed photons, regardless of their scattering status; therefore, PAM can penetrate more deeply by taking advantage of diffuse photons. Although 3D photoacoustics imaging has been achieved before on tissue phantoms ${ }^{4}$, ex-vivo blood vessels ${ }^{5}$, and animal brains ${ }^{6}$, no results have been reported on imaging microvasculature in vivo.

Here we demonstrate the 3D capabilities of PAM through (1) the depth-independent imaging of a carbon fiber phantom; and (2) the volumetric imaging of subcutaneous blood vessels in vivo.

Due to the large NA of the spherically focused ultrasonic detector being used, the foal zone of PAM is very short when the central frequency of the transducer is high. Thus, lateral resolution is degraded outside of the focal zone. In order to have a correct representation of the volumetric morphology of the blood vessels, a depth-independent lateral

\footnotetext{
*1wang@tamu.edu; phone: 1979 847-9040; fax: 1979 845-4450; oilab.tamu.edu
} 
resolution must be achieved. Therefore, we employ a numerical method, called the virtual point-detector based synthetic aperture focusing technique (SAFT) ${ }^{7}$, to compensate for the degraded off-focus lateral resolution. Based on phantom tests, both the lateral and axial resolutions are insensitive to the distance between the object and the surface of the ultrasonic detector after the SAFT correction.

\section{EXPERIMENTAL SETUP AND IMAGE FORMATION}

The schematic diagram of the PPAM is shown in Fig. 1. We used a Nd:YAG laser (repetition rate: $10 \mathrm{~Hz}$; pulse width: $6.5 \mathrm{~ns}$; wavelength: $532 \mathrm{~nm}$ at second harmonic) pumped tunable dye laser as the irradiation source. Laser light (584 nm in this study) was delivered through an optical fiber and expanded by a conical lens to form an annular beam. Light was then weakly focused into the tissue with its focal region overlapping the ultrasonic focus coaxially. The optical illumination was donut-shaped with a dark center so that no strong PA signals were produced from the skin surface, which prevented reverberations from over-shadowing signals from deep structures. The energy density deposited on the skin surface was kept well within the ANSI safety limit in our spectral range $\left(20 \mathrm{~mJ} / \mathrm{cm}^{2}\right)^{8}$. An ultrasonic transducer (central frequency: $50 \mathrm{MHz}$; nominal bandwidth: 70\%; NA: 0.5) was employed to detect the generated PA waves, which were then digitized by an oscilloscope working at a $250-\mathrm{MHz}$ sampling rate (without signal averaging) after being amplified by a wide-band low noise amplifier. A motion controller, which was synchronized by the triggering signal from the Nd:YAG laser, also controlled the tunable dye laser to change the optical wavelength automatically during spectral measurements when necessary.

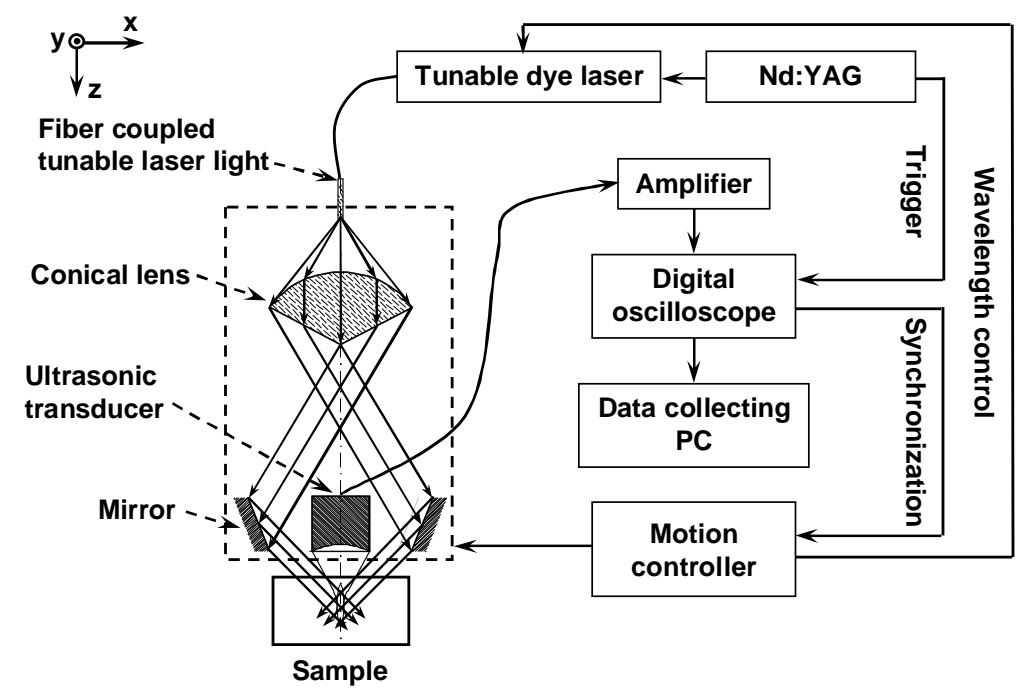

Fig. 1. Schematic of the PAM system.

To form an image, the PA waves were recorded at each transducer location for $2 \mu \mathrm{s}$. According to their time-ofarrival, the one-dimensional (1D) PA signals were converted into 1D depth-resolved images based on the sound velocity in soft tissue $(1.54 \mathrm{~mm} / \mu \mathrm{s})$. Then, raster scanning of the components in the dashed box in Fig. 1 in the $x-y$ plane produced a volumetric image. Based on the point spread function, which was acquired by imaging a carbon fiber with a diameter of $6 \mu \mathrm{m}$, the lateral and axial resolutions of the PAM were quantified to $45 \mu \mathrm{m}$ and $15 \mu \mathrm{m}$, respectively. The maximum imaging depth of PAM was measured to be more than $3 \mathrm{~mm}$ in live animals.

\section{RESULTS AND DISCUSSION}

\subsection{Imaging of carbon fiber phantom}

Five carbon fibers with a diameter of $6 \mu \mathrm{m}$ were placed at different heights within a range of $2 \mathrm{~mm}$ in an optical scattering solution to mimic micro blood vessels in biological tissues. A volume of $1 \times 4 \times 3 \mathrm{~mm}^{3}$ was imaged with a 20 $\mu \mathrm{m}$ scanning step. Based on the performance test of the 50-MHz ultrasonic transducer, the focal zone was about $0.5 \mathrm{~mm}$. 
Therefore, some carbon fibers were out of the focal region and, as a result, degraded lateral resolution was observed. Then, the SAFT was applied to correct the degraded lateral resolution at locations out of the focal region.

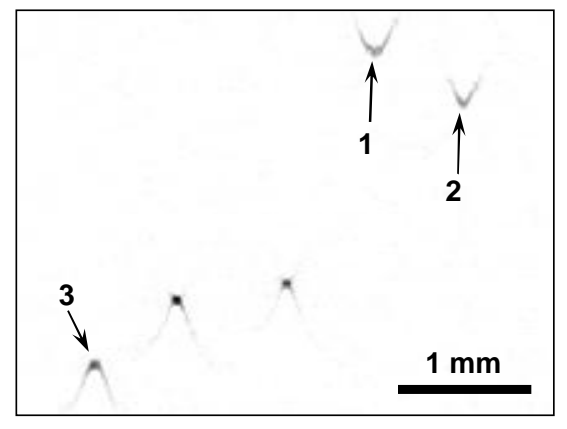

(A)

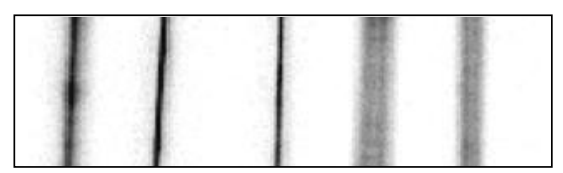

(C)

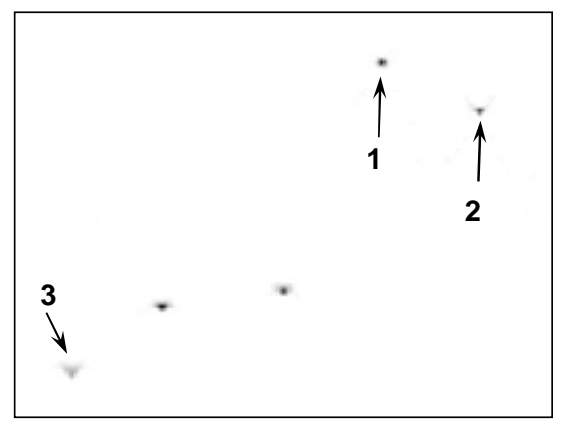

(B)

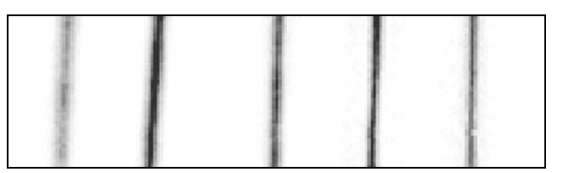

(D)

Fig 2. Images of a carbon-fiber phantom. Two cross-sectional images are shown to demonstrate the depthindependent resolution before (A) and after (B) SAFT correction. The comparison of the maximum-intensityprojection images before and after the SAFT correction is given by (C) and (D).

Figure 2 shows the carbon-fiber phantom results in two dimensions. Fig. 2(A) and Fig. 2(B) are typical crosssectional images before and after the SAFT correction. In Fig. 2(B), the fibers marked by 1, 2 and 3, which are strongly degraded in Fig. 2(A), have been corrected to a size that is comparable with those around the focal region. Fig. 2 (C) and Fig. 2 (D) are maximum-intensity-projection images along the $z$ axis, where the comparison of the degraded lateral resolution and correction is better visualized. This phantom study demonstrates that depth-independent spatial resolution, which is critical for the complete 3D imaging of blood vessels, can be achieved.

\subsection{Imaging of subcutaneous microvasculature in vivo}

The in-vivo imaging of subcutaneous blood vessels was conduced in a Sprague Dawley rat with a body weight of $200 \mathrm{~g}$. The hair at the scanning area on the back was removed before data acquisition. A dose of $87 \mathrm{mg} / \mathrm{kg} \mathrm{Ketamine,} \mathrm{plus}$ Xylasine $13 \mathrm{mg} / \mathrm{kg}$, was administered intramuscularly to anesthetize the rat, and supplemental injections $(\sim 50$ $\mathrm{mg} / \mathrm{kg} /$ hour) kept the rat motionless throughout the experiment. During the experiment, a Pulse Oximeter $(8600 \mathrm{~V}$, Nonin Medical, MN) was used to monitor the arterial blood oxygenation and heart rate; the body temperature was maintained throughout the experiment.

A 3D image was acquired at the 584-nm optical wavelength. An area of $10 \times 10 \mathrm{~mm}^{2}$ was scanned with a step size of $50 \mu \mathrm{m}$ along both the $x$ and $y$ axes. After being corrected by the SAFT, the skin signal was removed in order to better visualize the subcutaneous blood vessels. Due to motion artifacts, only an one-dimensional SAFT correction was performed along the $x$ axis. The final 3D image was volumetrically visualized using ray tracing.

Figure 3 shows the final result of the in-vivo experiment. The high spatial resolution of PAM imaging is demonstrated. Microvessels with diameters of less than 50 (occupying a single pixel) are well visualized. Due to the strong optical absorption of hemoglobin, a clean background has been achieved, which demonstrates the high contrast of PAM in imaging vasculature. Moreover, in the absence of a high concentration of melanin, the hemoglobin dominates the optical absorbers, and PAM achieves very high specificity in imaging the microvasculature. In Fig. 3, the two blood vessels marked by 1 and 2 can be observed at different depths below the skin surface, and the small vessels marked by 3 show different spatial orientations than 1 and 2. 
Volumetric PAM imaging of microvasculature with high spatial resolution, high contrast, and high specificity is invaluable for, for example, the study of tumor angiogenesis. The cancerous vessels can be observed at an early stage when their diameters are less than $20 \mu \mathrm{m}$. Complete volumetric information can be acquired at different stages to monitor tumor growth. Moreover, by combining volumetric imaging with spectroscopic measurement, functional information, such as hemoglobin oxygen saturation, can be acquired in 3D for in-depth physiological study on a single vessel basis.

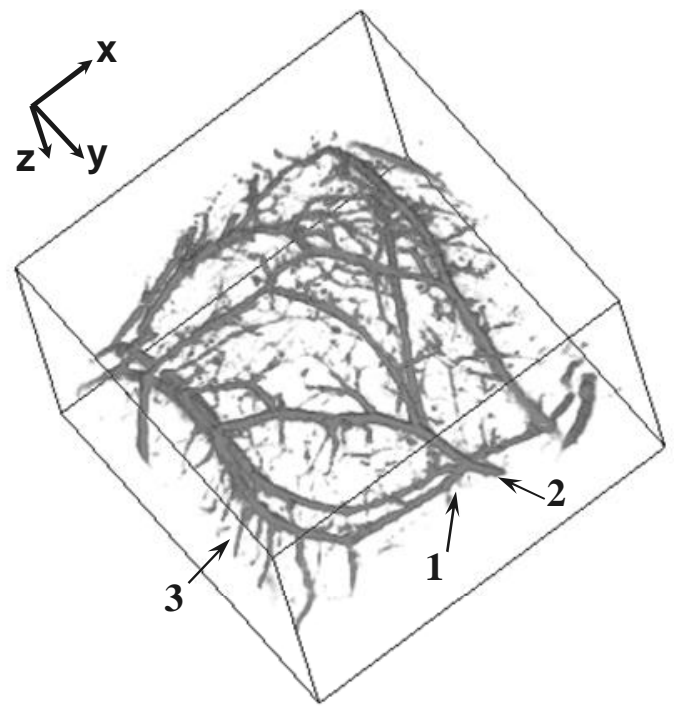

Fig. 3. 3D imaging of subcutaneous microvasculature in a small animal in vivo.

\section{SUMMARY}

In summary, 3D imaging of subcutaneous microvasculature has been achieved in small animals in vivo with high spatial resolution, high contrast and high specificity. The degraded lateral resolution at the off-focus region has been corrected by using the SAFT. After correction, depth-independent resolution has been reached and volumetric imaging can, therefore, correctly reflect the vessel structures. By combining volumetric imaging with spectroscopic measurement, 3D functional imaging can be accomplished.

\section{ACKNOWLEDGES}

We thank Ovidiu Craciun, Geng Ku, and Gina Lungu for experimental assistance. This project is sponsored by National Institutes of Health grants R01 EB000712 and R01 NS46214.

1 K. Maslov, G. Stoica, and L. V. Wang, "In vivo dark-field reflection-mode photoacoustic microscopy," Opt. Lett. 30, 625-627 (2005).

2 T. Sun, and G. J. Diebold, “Generation of ultrasonic waves from a layered photoacoustic source," Nature 355, 806-808 (1992).

3 F. A. Duck, Physical Properties of Tissue, Academic Press, London, 1990.

4 C. G. A. Hoelen, F. F. M. de Mul, R. Pongers, and A. Dekker, "Three-dimensional photoacoustic imaging of blood vessels in tissue," Opt. Lett. 23, 648-650 (1998).

5 M. C. Pilatou, N. J. Voogd, F. F. M. de Mul, and W. Steenbergen, "Analysis of three-dimensional photoacoustic imaging of a vasculature tree in vitro," Review of Scientific Instruments 74, 4495-4499 (2003).

6 X. Wang, Y. Pang, G. Ku, G. Stoica, and L. V. Wang, "Three-dimensional laser-induced photoacoustic tomography of mouse brain with the skin and skull intact," Opt. Lerr. 28, 1739-1741 (2003).

7 M.-L. Li, H. F. Zhang, K. Maslov, G. Stoica, and L. V. Wang, "Improved in-vivo photoacoustic microscopy based on a virtual detector concept," Opt. Lett. in press, (2006).

8 American national standard for the safe use of lasers Z136.1, American National Standards Institute, New York, 2000. 